\title{
Continuous Improvement of Quality Management in the Fall Prevention Process for Inpatients: a Historically Controlled Study
}

Hua XU

the Affiliated Brain Hospital of Nanjing Medical University

Xiang Cao

the Affiliated Brain Hospital of Nanjing Medical University

Yanhong ZHANG ( $\nabla$ zhangyanhong6312@126.com )

the Affiliated Brain Hospital of Nanjing Medical University

Zhaohong Chen

the Affiliated Brain Hospital of Nanjing Medical University

\section{Research Article}

Keywords: nursing, monitoring form, falls prevention, implementation, incidence rate, safety management

Posted Date: June 29th, 2021

DOI: https://doi.org/10.21203/rs.3.rs-612297/v1

License: (c) (i) This work is licensed under a Creative Commons Attribution 4.0 International License. Read Full License 


\section{Abstract}

Background: Falls are one of the most common adverse nursing events and sensitive indicators to measure nursing quality. The interventions for fall prevention are important; however, monitoring tools for the implementation are scarce.

Methods: To explore the effects of monitoring the fall prevention process in reducing inpatients' falls. Historically controlled trial and retrospective analysis. Explore and refer to the "structure-process-result" quality evaluation model. The design included assessment of fall risks, knowledge of prevention, participation of patients and their families, environment and management. The monitoring form for the implementation of fall prevention measures was used to monitor the effect and reduce the incidence rate of falls.

Results: The incidence rate of falls among inpatients with different risk levels was compared and analyzed before monitoring (control group) and 12 months, 24 months, 36 months later after monitoring. The trend chi-square results showed that the incidence rate of falls decreased from $0.199 \%$ o to $0.101 \%$, and the difference was statistically significant $(p<0.05)$. The probability of falls 12 months and 36 months later after intervention was 0.84 times and 0.51 times that of control group. The proportion of patients at high risk of falls had a decreasing trend.

Conclusions: After the use of the monitoring form, the incidence rate of falls reduces, nurses' professional quality and personal ability are improved, the awareness of patients and family members participating in patient's safety is strengthened, the environmental safety management is strengthened and the hardware facility is improved.

\section{Introduction And Background}

Falls refer to an unforeseen and involuntary change in posture owing to several risk factors, loss of equilibrium, and dropping on the ground or to a lower level [1]. It is one of the most frequently observed adverse nursing events. According to recent research [2], the incidence rate of inpatient falls is 6.63 falls/1000 patient days, of which $30-$ $50 \%$ of patients get injuries and $1-3 \%$ of patients suffer fractures. It not only prolongs the length of hospitalization and increases medical costs but also causes medical disputes [3]. Falls as a global public safety issue has gained attention from the international community. The National Patient Safety 2019 issued by the Joint Commission of the United State lists "prevention of falls" as one of the patient safety goals. In China, falls have also been regarded as one of the sensitive indicators to measure the quality of nursing[4].

Previous studies have shown that the history of falls [5], the activity of daily living dependence, and cognitive impairment $[5,6]$ are the high-risk factors of falls. Taking antihypertensive diuretics, anti-Parkinson's drugs, hypnotic drugs [7], antipsychotics, and antidepressants [8] may also be important factors of falls. Many complex factors affect the risk of falls. Recently the management decisions of preventing patients from falling mainly include two points. The first is by predicting the risk of the falls in patients. The second is by administering preventive measures based on the predicted risk factors. These measures are in accordance with that reported by Yi Yanzhi[9]. Currently, various falls risk assessment tables have been designed for different inpatients, which are also the most widely used and researched methods employed to predict falls worldwide. However, its sensitivity is debatable.

To date, the interventions for fall prevention comprise those of environment, education, communication, and nursing procedures [10]. Adverse environmental factors may lead to elderly falls, escalating their injuries [11]. China 
has promulgated the standard such as Barrier-free and Architecture Design Codes in the General Hospital. It has some requirements about the design of wards, toilets, corridors, and lighting. However, there are still some hospitals that cannot fulfill the basic conditions aforementioned or fail to renovate the environment in time. A randomized study involving 1822 patients with a high risk of falls demonstrated that professionally-trained nurses could significantly reduce the occurrence of falls [12]. Therefore, training and education for nursing staff can enhance their knowledge [13], and is one of the critical factors of reducing the risk of patients' falls [14]. However, to date, China has not set up courses in patient safety education. Simultaneously, there is little training for falls and related education projects. Lawton[15]found that encouraging patients to participate in safety management is of great significance for preventing falls. Medical organizations have proposed that patients should be encouraged to participate in self-safety to avoid adverse events and ensure a fundamental guarantee to self-welfare. Patients should self-supervise and participate actively to maintain their safety. Although nurses play an important role in the management of patient safety, they cannot replace the effect of patients' self-participation[16, 17].

Studies have shown that devices based on new sensor technology such as inertial/pressure sensors and depthsensing cameras are used to assist in monitoring the risk of fall prevention [18]. For example, smart mattresses using sensors can monitor hazardous behaviors, including patients' movements [19]. Anti-drop shoes [20] equipped with lasers can detect obstacles in advance and give an alarm to remind the elderly to consider obstacles in front of them. There are some wearable devices with airbags, which can reduce damage after falls [21]. Meanwhile, with the continuous development and maturity of virtual reality, the occurrence of falls may be reduced [22], and new ideas and solutions for clinical practice may be provided.

Thus, researchers worldwide have made significant efforts, and managers have applied a variety of management models to prevent falls. Moreover, China has made remarkable progress. The main measures of preventing falls are the risk factor interventions that affect falls, the use of fall risk tools to screen and assess, personalized education manuals and warning signs, and the invention of new technologies. However, the tools for evaluating the effectiveness of the implementation of various measures are scarce. In early 2017, to perform nursing control of inpatients' falls adequately and ensure the implementation of safety management measures, our nursing experts designed and optimized monitoring form for fall prevention. The clinical effect was satisfactory. The report is as follows.

\section{Method}

\subsection{Setting and participants}

A total of 391,286 patients were admitted to each clinical nursing unit daily. The falls that occurred between 2017 and 2019 (12 months, 24 months, 36 months later after intervention )were selected as the intervention group, while 78 inpatient falls that occurred in 2016 were selected as the control group. A total of 78 cases of inpatient falls occurred, among which 54 cases were rated as high risk. In 2017, there were 407,321 inpatients and 68 cases of falls, while there were 415,456 inpatients and 49 cases of falls in 2018 . There were 416,830 inpatients and 42 cases of falls in 2019. For 2017, 2018, and 2019, 40, 23, and 18 cases, respectively, were rated as high risk.

\subsection{Design and optimization of the monitoring form \\ 2.2.1 Refer to "structure-process-result" three-dimensional model[23]to construct monitoring form}


Based on the principle of easy understanding, simple scoring, and high applicability, the expert team of quality special inspection form for falls prevention comprised two directors of the nursing department, three head nurses of the psychiatric department, five head nurses from the department of neurosurgery, outpatient and emergency, critical care medicine, and geriatric neurology. The expert team also included one core nurse with intermediate and senior titles in each department. Based on "structure-process-result" quality evaluation model[23], the Clinical Practice Guidelines for Inpatient Falls Prevention [24] as suggested by the China Health and Family Planning Commission, Medical Quality Management Measures [25], Chinese Hospital Association Patient Safety Target (2017 edition), domestic and foreign literature, the risk factors of inpatient falls during hospitalization, data support, clinical practices, and group discussions, the preliminary version of monitoring form for falls prevention was formulated. After two rounds of clinical application in 3 months, multiple feedbacks, and discussions, the final version was achieved. The data was entered into the nursing management information platform and tablet personal computer (PC) terminal. The members of quality control and nursing managers at all levels could achieve evaluation and guidance of fall prevention using tablet PC. It made nursing management paperless and scientific.

\subsubsection{Introduction of monitoring form}

The final monitoring form for fall prevention comprised a table head, six quality control items, rules for points deduction, points of each item, and existing problems. The six quality control items were as follows: खThe assessment methods of falls and basis for scoring; $『$ Correct falls assessment scale and information about falls; هDiversified health education methods; 『Environmental facility; $₫$ Participation of patients/family members/caregivers'; and $₫$ Measures of management of falls prevention, as shown in Table 1. 
Table 1

Quality special inspection form for falls prevention

\section{Quality special inspection form for falls prevention}

$\begin{array}{lll}\text { Module group } & \begin{array}{ll}\text { Module } \\ \text { name: }\end{array} & \begin{array}{l}\text { Inspection } \\ \text { standard } \\ \text { of falls } \\ \text { prevention }\end{array} \\ & \text { predule level }\end{array}$

(V2017)

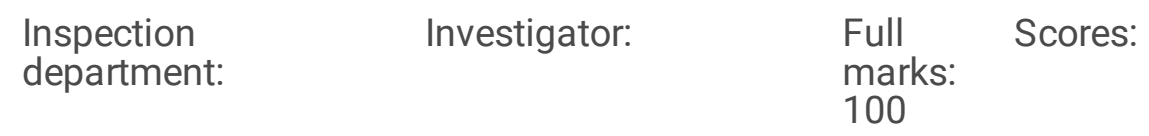

$\begin{array}{ll}\text { Be investigated } & \text { Be investigated } \\ \text { (Patient): } & \text { (Nurse): }\end{array}$

\begin{tabular}{|c|c|c|c|c|c|}
\hline content & $\begin{array}{l}\text { Points deduction } \\
\text { rules }\end{array}$ & Scores & Deduction & $\begin{array}{l}\text { Existing } \\
\text { problem }\end{array}$ & $\begin{array}{l}\text { Continuous } \\
\text { improvement }\end{array}$ \\
\hline
\end{tabular}

1.Nurses know the 2 points deducted for 2

assessment knowing falls

methods of falls, assessment method

master the scoring less than $60 \%, 1$

basis of the fall point deducted less

assessment scale, than $80 \%$

the risk factors of

falls, correctly judge the risk of falls, know the patients at high risk, and know the preventive measures for falls. (On-site inspection, assessment and interview)

2 points deducted for

2 knowing falls scoring basis less than $60 \%$, 1 point deducted less than $80 \%$

2 points deducted for 2 knowing falls risk factor of patients less than $60 \%, 1$ point deducted less than $80 \%$

2 points deducted if
degree of falls risk is
judged correctly less
than $60 \%, 1$ point
deducted less than
$80 \%$

2 points deducted for knowing high-risk patients less than $60 \%, 1$ point deducted less than $80 \%$

4 points deducted for knowing falls prevention measures less than $60 \%, 1$ point deducted less than $80 \%$ 
Quality special inspection form for falls prevention

2.Correctly use the appropriate falls assessment scale. Apply communication methods to conduct risk assessment for patients who are newly admitted, transferred, operated, have diseases changes and hospitalized for a long time. Correctly collect information related to patients' falls. The original score of the evaluation form is contained in the electronic medical record has original score of assessment scale. The risk score should be reflected in the nursing record. (On-site inspection, assessment and interview)
Falls scale selected $\quad 2$

is inappropriate.

2 points deducted for 2 knowing $60 \%$ of content in evaluation using communication methods, 1 point deducted less than $80 \%$

4 points deducted for

4 the correct use of falls assessment form less than $60 \%$, 2 points deducted less than $80 \%$

The patients who 2 were newly admitted, transferred, operated, had disease changes or hospitalized for a long time were not evaluated.

2 points deducted if 2 the information of patients' falls is less than $60 \%, 1$ point deducted if less than $80 \%$.

No original score of

3.Apply diversified health education methods to provide health guidance of falls prevention, patients/family members/caregivers know falls prevention knowledge(on-site inspection, interview) falls assessment from scale

Falls assessment score is missing in the nursing record.

Single health
education method

Nurses not guide 1

patients' getting up

Nurses do not guide family members' getting up

Nurses not caregivers' getting up

High risk patients lacking personalized education content

2

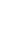

1

1

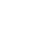

Nurses do not

instruct the way of

calling for patients 
Quality special inspection form for falls prevention

Nurses do not

1

instruct they way of

seeking help

Nurses do not tell the

1

patients not to cross

the bed bar

Nurses do not

1

instruct the correct

way of moving

Nurses do not

1

instruct the correct

way of getting in and

out of bed

Nurses do not

1

instruct the way of

using a urinal in bed

Nurses do not

instruct the way of

using bed pan in bed

4 points deducted for 4

patients/family

members/caregivers

who are aware of

falls prevention

measures less than

$60 \%, 2$ points

deducted less than

$80 \%$

No health education

related to falls

4.Environmental facilities safety(onsite inspection)
No warning signs of

"caution slipping" in

the ward

No bed bar

Bed bars are too short of wobbly

The daily use of bedside table is not convenient for patients to assess

The handle of bed is not tucked

The floors are wet in wards, corridors, and toilets

The light in the wards, corridors, and toilets are too dark.
1

2

1

2

2

2

2

2

\section{2}




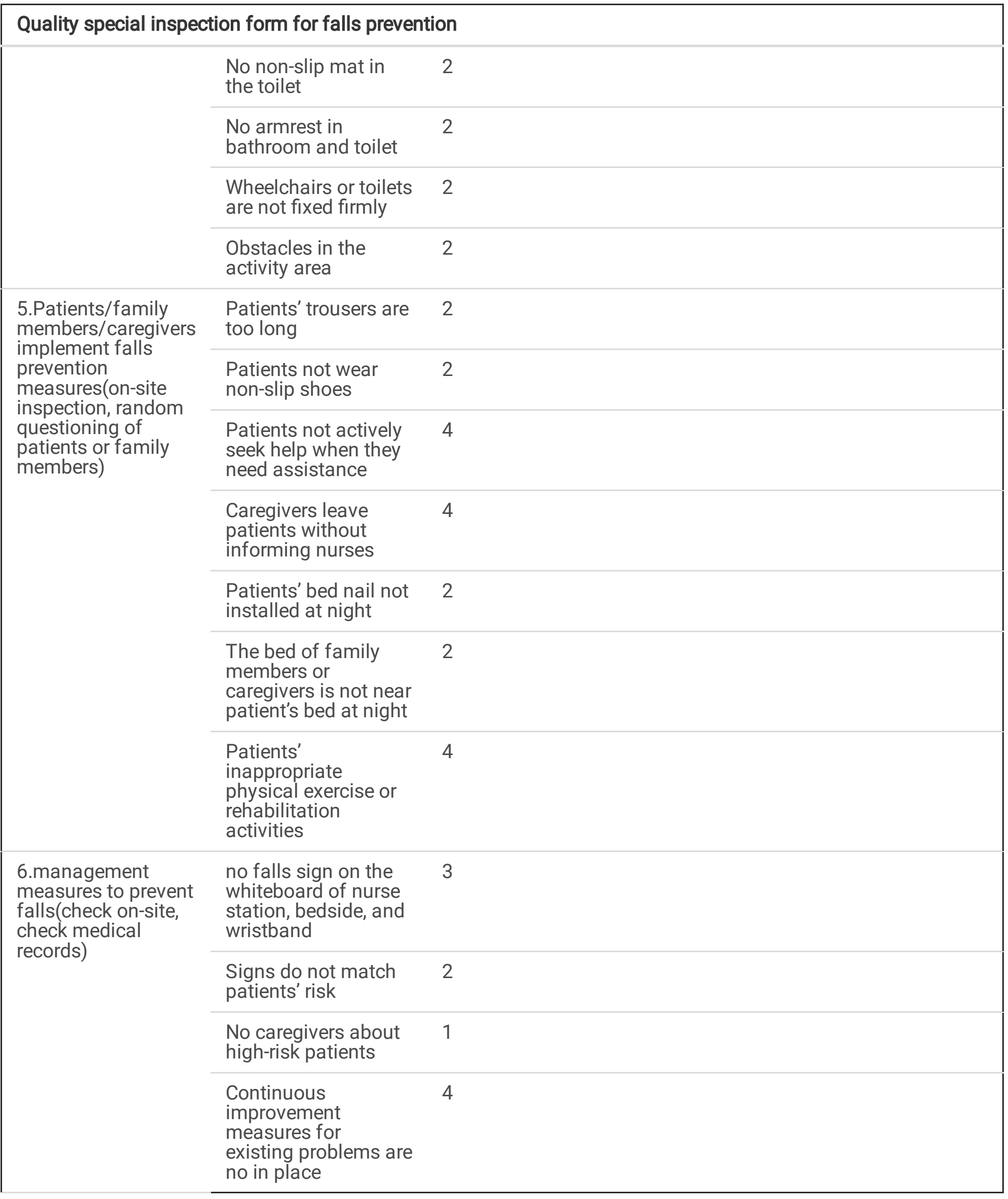

\subsubsection{Unification and use of the monitoring form}


The head nurse of each ward logged in their nursing management platform, printed the monitoring form, and conducted investigation and assessment on the implementation effect of fall prevention measures. The head nurse of the department selected two nurses of different levels to examine the use of the fall risk assessment scale and health education for fall prevention. They rectified the problems in the above inspection and assessment sequentially and reported them to the nursing department for record.

\subsection{Clinical application}

\subsubsection{Set up nursing safety quality control team}

A three-level nursing quality management system was established considering "nursing department-departmentward." Meanwhile, a nursing safety team was instituted. The director of the nursing department was the team leader, while the head nurse was the vice team leader. The team members comprised the head nurse of each ward and 1-2 senior nurses. Also, the core members of the nursing safety quality control team included four group leaders and two secretaries who were selected by the vice team leader based on their professional titles, ability levels, and the nature of the department. At the beginning of each year, the safety training and activities were formulated based on the analysis of former adverse events and potential safety hazards. They were responsible for the training and supervision of nursing safety team members' awareness of fall risks and safety management. The nurses had a high professional ability. Based on the model "the old leads the new," the old nurses could guide and train the new nurses in the ward to make them self-reliant promptly.

\subsubsection{Improve the falls management system}

\A non-punishable adverse event reporting system was established. The nurses were encouraged to take the initiative to report adverse events to the head nurse of the ward, department, and nursing department within $2 \mathrm{~h}$ after the falls occurred. In the ward, the falls were analyzed, discussed, and corrective measures were proposed and followed up. The nursing adverse event was reported to the management platform within two working days. $\otimes T h e$ adverse event of falls was shared. The nursing department organized a meeting to share the quarterly adverse events. The head nurse analyzed the rationale for falls in the ward, shared the corrective measures, and tracked results. The nursing safety group conducted sequential analysis on falls every 3,6, and 12 months, and proposed corrective measures for common problems, and conducted analysis on typical cases. $\otimes T$ The falls management was continuously improved. Based on the results of each round of quality control and inspection of falls, the ward improved the falls management system and process and reported it to the nursing department, and formulated the "Improved Hendry II Falls Risk Model," “Non-psychiatric Falls Prevention Management System,” and "Classification Management System for Falls Prevention." According to specialized diseases and patients' falls characteristics, the nursing theory knowledge, health education manuals, and videos were upgraded, such as the revised "Measures for Preventing Falls in the Ward." The ward with a high incidence rate of falls conducted quality control circles and plan-do-check-act (PDCA). $\triangle$ The nursing department organized a joint meeting with the departments of medicine, general affairs, equipment, pharmacy, and accompanying management to discuss preventive measures and for the implementation of the security system for falls.

\subsubsection{Organize training on the knowledge and skills of falls prevention}

Currently, our hospital uses three types of falls risk assessment scales, namely, the modified version of Hendry II Falls Risk Model [26], Chinese version of Morse Falls Evaluation Scale [27], and Falls Risk Assessment Table. The appropriate fall risk assessment scale was selected based on the disease type and the patient characteristics. The 
nurses were trained regarding the risk assessment for fall prevention, knowledge and skills, management system, and emergency procedures. The above contents were evaluated. Combining the ward characteristics, the health education manuals, videos, and falls prevention warning signs would be formulated. The nursing safety team assisted the nursing department in performing the theory courses of analyzing fall-related risk factors, preventive measures, nursing safety knowledge, and law. The activity of falls was held twice a year, including falls nursing health education scene simulation game, emergency treatment of falls, publicity videos, and micro-class competitions. The activity was achieved by senior and junior nurses in each ward. This aimed to enable medical staff to have an "immersive experience," which enhanced the awareness and ability of warning and management of falls.

\subsubsection{Special inspection and continuous improvement}

The monitoring form for fall prevention was applied by the nursing department at three levels. The head nurse was responsible for self-inspection and reported monthly potential safety hazards. The fall prevention measures were implemented in the ward with a high incidence fall rate quarterly by a three-level security team of the nursing department and by the special quality control team comprising senior nurses of the nursing safety group every six months. The effect of fall prevention measures or whether receiving health education was implemented by on-site inspection, random inquiry of patients, family members, or caregivers was assessed. The results of each quality control inspection would be uploaded to the nursing management platform. After each round of performing quality special inspection for fall prevention, the head nurse of each ward would analyze the etiology, make rectification plans, implement rectification measures, identify the responsible persons and deadlines, and focus on problems and implementation in the next round of inspection. Implementing the PDCA cycle played an important role in the continuous improvement of the nursing quality of fall prevention. The nursing department conducted data analysis using the management platform and revised the procedures, measures, and prevention systems related to falls prevention.

\subsection{Statistical analysis}

The incidence rate of falls among inpatients and rate of falls among patients with different risk scores were compared between control group (2016) and intervention group(2017 to 2019) 12 months, 24 months, 36 months later using a monitoring form for fall prevention. SPSS 22.0 software was used to process data. $\square^{2}$ test was used, and $\mathrm{P}<0.05$ indicated a statistically significant difference.

\section{Results}

The annual incidence rate of patient falls was analyzed using trend chi-square, and the results showed that the incidence rate of control group and intervention group decreased year by year from 2016 to $2019(P<0.05)$, as shown in Table 2. 
Table 2

Trend analysis of patients' falls rate from 2016 to 2019

\begin{tabular}{|llllll|}
\hline Year & Beds & Falls & Incidence & $\square^{2}$ data & $P$ \\
& per day & times & Rate (\%o) & & \\
\cline { 1 - 4 } 2016 & 391286 & 78 & 0.199 & 16.509 & 0.000 \\
2017 & 407321 & 68 & 0.167 & & \\
2018 & 415456 & 49 & 0.118 & & \\
2019 & 416830 & 42 & 0.101 & \\
\hline
\end{tabular}

The incidence rate of falls before the intervention (2016) was considered as a reference, and that after the intervention (2017-2019) was analyzed using the chi-square test. The results showed that compared with control group, there was no statistically significant difference after 12 months of intervention $(2017)(P>0.05)$. However, two groups dropped 16\%. Comparing with control group, after 24 months and 36 months of intervention (2018 and 2019), the incidence rate of falls was statistically significant $(P<0.05)$, with relative risk 0.59 (95\% confidence interval: 0.41-0.85), $0.51(0.35-0.74)$, as shown in Table 3.

Table 3

Comparison and analysis of patients' fall rate from 2016 to 2019

\begin{tabular}{|c|c|c|c|c|c|c|c|c|}
\hline \multirow[t]{2}{*}{ Year } & \multirow{2}{*}{$\begin{array}{l}\text { Beds } \\
\text { per day }\end{array}$} & \multirow{2}{*}{$\begin{array}{l}\text { Falls } \\
\text { times }\end{array}$} & \multirow[t]{2}{*}{ Incidence Rate (\%o) } & \multirow[t]{2}{*}{$\square^{2}$ data } & \multirow[t]{2}{*}{$\mathbf{P}$} & \multirow[t]{2}{*}{ RR } & \multicolumn{2}{|l|}{ RR 95\%Cl } \\
\hline & & & & & & & Lower limit & Upper limit \\
\hline 2016 & 391286 & 78 & 0.199 & - & - & 1 & - & - \\
\hline 2017 & 407321 & 68 & 0.167 & 1.146 & 0.284 & 0.84 & 0.61 & 1.16 \\
\hline 2018 & 415456 & 49 & 0.118 & 8.483 & 0.004 & 0.59 & 0.41 & 0.85 \\
\hline 2019 & 416830 & 42 & 0.101 & 13.211 & 0.000 & 0.51 & 0.35 & 0.74 \\
\hline
\end{tabular}

The results of chi-square analysis demonstrated that the proportion of high, medium, low, and no risk of falls every year before and after the application of monitoring form was different $(P<0.05)$. Collectively, the proportion of high risk showed a downward trend, and the proportion of medium risk, low risk, and no risk had an upward trend, as shown in Table 4. 
Table 4

Analysis of fall rate in patients with different scores before and after intervention

\begin{tabular}{|c|c|c|c|c|c|c|c|}
\hline Year & $\begin{array}{l}\text { Falls } \\
\text { times }\end{array}$ & $\begin{array}{l}\text { High risk cases } \\
(\%)\end{array}$ & $\begin{array}{l}\text { Medium } \\
\text { risk cases } \\
(\%)\end{array}$ & $\begin{array}{l}\text { Low risk cases } \\
(\%)\end{array}$ & $\begin{array}{l}\text { No risk cases } \\
(\%)\end{array}$ & $\begin{array}{l}\text { Trend } \\
\square^{2} \text { data }\end{array}$ & $P$ \\
\hline 2016 & 78 & $54(69.2)$ & $16(20.5)$ & 8 (10.3) & $0(0.0)$ & \multirow[t]{4}{*}{12.944} & \multirow[t]{4}{*}{0.000} \\
\hline 2017 & 68 & $40(58.8)$ & $14(20.6)$ & $14(20.6)$ & $0(0.0)$ & & \\
\hline 2018 & 49 & $23(46.9)$ & $12(24.5)$ & $10(20.4)$ & $4(8.2)$ & & \\
\hline 2019 & 42 & $18(42.9)$ & $12(28.6)$ & $10(23.8)$ & $2(4.8)$ & & \\
\hline
\end{tabular}

\section{Discussion}

\subsection{The reduction of the incidence rate of falls using the monitoring form}

The monitoring form for fall prevention was simple, clear, and feasible for points deduction. It was applied for training, assessment, and quality control of falls management in various clinical departments, combined with a nursing information system and special falls quality control process. Based on this, the important sections of the monitoring form, which did not meet the standards, would be improved further. The results showed that the incidence rate of inpatient falls decreased from $0.199 \%$ in 2016 to $0.101 \%$ o in 2019 , a downward trend year by year. Analysis of the annual falls incidence rate showed that the probability of falls in 2017 and 2019 was 0.84 times and 0.51 times that in 2016. It had a remarkable effect on reducing the incidence rate of falls. After using the monitoring form for fall prevention, the proportion of high risk had a downward trend, and the proportion of medium risk, low risk, and no risk had an upward trend. The possible reasons were as follows. Nurses relied on the fall scoring scale and lack of consideration of influencing factors. खNurses did not pay attention to the implementation of measures for patients who were not at high risk of falls. $₫$ The patients and family members overestimated the patients' activity, exercise ability, and poor compliance with measures to prevent falls. The results of this study indicated that nurses should not rely excessively on the fall risk assessment form in the future. They should focus more on the patients with medium and low risks and strengthen the education and guidance. The previous research results showed [28] that the occurrence of falls was related to the patients' factors, disease, nurses' professional quality, facility environment, management, and other factors. The monitoring form designed for fall prevention, integrated these influencing factors. In each round of falls quality special inspection, PDCA analysis was performed on the existing problems, which assisted the nursing managers to strengthen nursing quality management system and quality. To ensure that the core nursing system was implemented completely, work procedures, rules and regulations were improved, nurses' advice was noted, the system and process were discussed, rectified, and revised, and clinical inspection and supervision were performed well. It raised the management level of nursing managers, modified the traditional analysis based on experience, and classified errors as personal factors.

\subsection{The use of the monitoring form improves nurses' professional quality and personal ability}

The research [29] showed that junior nurses are a high-risk group of adverse events. It may be possible that due to less experience or professional knowledge, their clinical workability, judgment, and communication skills were poor, which directly affected the quality of care and patients' safety. Therefore, nursing managers should pay more attention to the training and management of new and junior nurses. They should not only do a satisfactory job in 
"three basics" training but also strengthen safety awareness and establish safety beliefs. The assessment of fall risk [30] is the screening and evaluation of patients at high risk of falls and is the basis of safety management. It has become the basis for clinical nursing staff to take measures to prevent falls. Therefore, nursing staff should use the correct fall assessment scale for patients with high risk to improve the detection rate of those at risk of falls. When the patients' condition changes, medication adjustments should be re-assessed, and the nursing risk assessment should be modified [31]. Therefore, nursing managers should focus on the assessment of falls risk, strengthen the specialized theoretical knowledge of the nursing staff and strengthen the quality control of the nursing assessment. The training before the use of the assessment form, supervision during the use, and evaluation after the use can help nurses master the knowledge and nursing skills related to falls. It assists the nurses to discover high-risk factors and take practical interventions in time. In response to the problems that arise during the inspection, the nursing department, nursing safety group, and ward make plans to provide nurses with comprehensive knowledge and skills training, such as standardization of fall prevention and safety management systems, procedures, and health education. Creating a well-organized nursing safety culture atmosphere, cultivating nurses' risk awareness, prevention awareness, and legal awareness are the guarantee of reducing the occurrence of falls.

\subsection{The use of the monitoring form strengthens the awareness of patients and family members participating in patient's safety}

Elderly patients are at significantly high risk for falls [32]. The hospital in this study was conducted at a neurological and psychiatric center, where most of the inpatients were elderly. Due to the decline of physical ability, overestimating their exercising ability, and poor compliance with the implementation of fall prevention measures, the old patients decline to accept the assistance of family members or caregivers. For patients with poor compliance, nurses should adopt a customized manner to emphasize the importance and precautions of preventing falls [33]. According to patients' disease, cultural background, age, sex, characters, safety concepts, and the stopping elderly accidents, deaths, and injuries tool [34] issued by the United States Centers for Disease Control and Prevention, videos, mold materials, and micro-classes were utilized to advise patients and family members about the knowledge of falls prevention using simple words, pictures, and texts. The guidance for patients with high risk was strengthened, and the assessment results, fall risk factors, and preventive measures were informed to patients and their families in time, and they were inquired to sign for approval. Meanwhile, patients, family members, and caregivers were informed about the significance and importance of the fall warning sign, which was placed beside the patients' beds. The patients were forbidden to turn the warning sign on without authorization. The nurses marked the bed numbers with a high risk of falls on the whiteboard in the nurse station. The doctors marked and emphasized the importance of prevention to patients and their attendants again. This mode of participation is conducive to promote attention to falls prevention and improve their cooperation. It has created a culture of "everyone participating in patient safety management" and realized the transformation from traditional mode to the new mode. Briefly, effective prevention of falls requires the participation of doctors, nurses, patients, and their families, effective communication, understanding, and cooperation. It will establish the preventive measures and ensure patient's safety [35].

\subsection{After the use of the monitoring form, the environmental safety management is strengthened, and the hardware facility is improved.}

The variables related to falls indicate that in addition to the patients' factors, the falls are often related to the surrounding environment and hospital equipment, such as water on the floor, no anti-slip floor tiles, night light, no 
armrest in the bathroom. The toilet or bathroom is a high-risk place for falls [36]; therefore, our hospital has improved the facilities in the toilets, added armrests, sitting toilets, anti-slip floor tiles/anti-slip mats, installed emergency buttons, placed signs when the floor is slippery, and placed anti-slip mats to reduce environmental impact. The pager is placed within easy reach for patients to guide them to familiarize themselves with the ward environment. The night light is switched on, and obstacles around the patients are removed. The height of the bed is adjusted to ensure that the patient's feet are not suspended in air, while sitting on the edge of the bed. The nursing level is not the primary cause of falls. It requires the cooperation of related departments and multidisciplinary teams [37-39], which can reduce the incidence rate of falls and improve patients' satisfaction.

\section{Conclusions}

In summary, based on the three-level and nursing safety quality control team, this study utilized a monitoring form for prevention of falls, and comprehended the complete process of assessment, planning, implementation, and evaluation of clinical falls. Strengthening the safety knowledge by training the nurses, supervising the implementation of fall measures, mobilizing patients and family members participating in prevention of fall, and cooperating with various departments to rectify, can play a positive role in reducing falls. Therefore, the monitoring form may be applied clinically for preventing falls; thus, promoting patients and family members to participate actively in the patients' safety.

\section{Abbreviations}

No abbreviations.

\section{Declarations}

\section{Acknowledgements}

The authors would like to thank all the participants for their support.

\section{Funding}

No funding was received.

\section{Author contributions}

YZ and ZC conceptualised the idea. HX designed the study with review from $\mathrm{YZ}$ and ZC. HX and XC collected the data, $\mathrm{HX}$ and $\mathrm{YZ}$ analysed the data. All authors drafted components of the manuscript, all critically reviewed and approved the final draft.

\section{Availability of data and materials}

The datasets used and/or analyzed during the current study are available from the corresponding author on reasonable request.

\section{Ethical declarations}

\section{Ethics approval and consent to participate}


The procedures of this study were reviewed and approved by the Institutional Review Board of the Affiliated Brain Hospital of Nanjing Medical University (2017-KY089). All participants provided written informed consent before participating in the study.

\section{Consent for publication}

Not applicable.

\section{Competing interest}

The authors have no competing conflict.

\section{References}

1. Grossman DC, Curry SJ, Owens DK, Barry MJ, Caughey AB, Davidson KW, Doubeni CA, Epling JJ, Kemper AR, Krist $\mathrm{AH}$ et al.Interventions to Prevent Falls in Community-Dwelling Older Adults: US Preventive Services Task Force Recommendation Statement.JAMA.2018;319(16):1696-1704.Available from:http://dx.doi.org/10.1001/jama.2018.3097.

2. Morris R, O'Riordan S.Prevention of falls in hospital.Clin Med (Lond).2017;17(4):360-362.Available from:http://dx.doi.org/10.7861/clinmedicine.17-4-360.

3. Zhao YL, Bott M, He J, Kim H, Park SH, Dunton N.Multilevel Factors Associated With Injurious Falls in Acute Care Hospitals.J NURS CARE QUAL.2018;33(1):20-28.Available from:http://dx.doi.org/10.1097/NCQ.0000000000000253.

4. TANG L W,YE Z H,PAN H Y.Construction of a nursing sensitive quality indicators system [J].Chinese Journal of Nursing,2013,48(9):801-803.

5. Hayakawa T, Hashimoto S, Kanda H, Hirano N, Kurihara Y, Kawashima T, Fukushima T.Risk factors of falls in inpatients and their practical use in identifying high-risk persons at admission: Fukushima Medical University Hospital cohort study.BMJ OPEN.2014;4(8):e5385.Available from:http://dx.doi.org/10.1136/bmjopen-2014005385.

6. Kvelde T, Lord SR, Close JC, Reppermund S, Kochan NA, Sachdev P, Brodaty H, Delbaere K.Depressive symptoms increase fall risk in older people, independent of antidepressant use, and reduced executive and physical functioning.Arch Gerontol Geriatr.2015;60(1):190-195.Available from:http://dx.doi.org/10.1016/j.archger.2014.09.003.

7. Shuto H, Imakyure O, Matsumoto J, Egawa T, Jiang Y, Hirakawa M, Kataoka Y, Yanagawa T.Medication use as a risk factor for inpatient falls in an acute care hospital: a case-crossover study.Br $\mathrm{J}$ Clin Pharmacol.2010;69(5):535-542.Available from:http://dx.doi.org/10.1111/j.1365-2125.2010.03613.x.

8. Deandrea S, Bravi F, Turati F, Lucenteforte E, La Vecchia C, Negri E.Risk factors for falls in older people in nursing homes and hospitals. A systematic review and meta-analysis.Arch Gerontol Geriatr.2013;56(3):407415.Available from:http://dx.doi.org/10.1016/j.archger.2012.12.006.

9. Yi Y Z., Shannon H. Houser., Shi L P., et al. Predictive analysis of fall risk factors in inpatients (J). Nursing Research, 2019,33(09):1593-1596.

10. Zhao YL, Bott M, He J, Kim H, Park SH, Dunton N.Evidence on Fall and Injurious Fall Prevention Interventions in Acute Care Hospitals.J Nurs Adm.2019;49(2):86-92.Available from:http://dx.doi.org/10.1097/NNA.0000000000000715. 
11. Donald IP, Pitt K, Armstrong E, Shuttleworth H.Preventing falls on an elderly care rehabilitation ward.CLIN REHABIL.2000;14(2):178-185.Available from:http://dx.doi.org/10.1191/026921500677888641.

12. Ang E, Mordiffi SZ, Wong HB.Evaluating the use of a targeted multiple intervention strategy in reducing patient falls in an acute care hospital: a randomized controlled trial.J ADV NURS.2011;67(9):1984-1992.Available from:http://dx.doi.org/10.1111/j.1365-2648.2011.05646.x.

13. Liu H, Shen J, Xiao LD.Effectiveness of an educational intervention on improving knowledge level of Chinese registered nurses on prevention of falls in hospitalized older people--a randomized controlled trial.Nurse Educ Today.2012;32(6):695-702.Available from:http://dx.doi.org/10.1016/j.nedt.2011.09.009.

14. Tricco AC, Thomas SM, Veroniki AA, Hamid JS, Cogo E, Strifler L, Khan PA, Sibley KM, Robson R, MacDonald $\mathrm{H}$ et al.Quality improvement strategies to prevent falls in older adults: a systematic review and network metaanalysis.AGE AGEING.2019;48(3):337-346.Available from:http://dx.doi.org/10.1093/ageing/afy219.

15. Lawton R, O'Hara JK, Sheard L, Armitage G, Cocks K, Buckley H, Corbacho B, Reynolds C, Marsh C, Moore S et al.Can patient involvement improve patient safety? A cluster randomised control trial of the Patient Reporting and Action for a Safe Environment (PRASE) intervention.BMJ QUAL SAF.2017;26(8):622-631.Available from:http://dx.doi.org/10.1136/bmjqs-2016-005570.

16. Vaismoradi M, Jordan S, Kangasniemi M.Patient participation in patient safety and nursing input - a systematic review.J CLIN NURS.2015;24(5-6):627-639.Available from:http://dx.doi.org/10.1111/jocn.12664.

17. Wright J, Lawton R, O'Hara J, Armitage G, Sheard L, Marsh C, Grange A, McEachan R, Cocks K, Hrisos S et al: Improving patient safety through the involvement of patients: development and evaluation of novel interventions to engage patients in preventing patient safety incidents and protecting them against unintended harm. Southampton (UK): NIHR Journals Library; 2016.

18. Sun R, Sosnoff JJ.Novel sensing technology in fall risk assessment in older adults: a systematic review.BMC GERIATR.2018;18(1):14.Available from:http://dx.doi.org/10.1186/s12877-018-0706-6.

19. Balaguera HU, Wise D, Ng CY, Tso HW, Chiang WL, Hutchinson AM, Galvin T, Hilborne L, Hoffman C, Huang CC et al.Using a Medical Intranet of Things System to Prevent Bed Falls in an Acute Care Hospital: A Pilot Study.J MED INTERNET RES.2017;19(5):e150.Available from:http://dx.doi.org/10.2196/jmir.7131.

20. Lin TH, Yang CY, Shih WP.Fall Prevention Shoes Using Camera-Based Line-Laser Obstacle Detection System.J HEALTHC ENG.2017;2017:8264071.Available from:http://dx.doi.org/10.1155/2017/8264071.

21. Li H Q., Ning Y K., Yang J F., et al. Study on wearable fall protective aribag [J]. Integrated technology, 2018,7(02):69-77.

22. Mirelman A, Rochester L, Maidan I, Del DS, Alcock L, Nieuwhof F, Rikkert MO, Bloem BR, Pelosin E, Avanzino $L$ et al.Addition of a non-immersive virtual reality component to treadmill training to reduce fall risk in older adults (V-TIME): a randomised controlled trial.LANCET.2016;388(10050):1170-1182.Available from:http://dx.doi.org/10.1016/S0140-6736(16)31325-3.

23. Donabedian A.Quality assurance. Structure, process and outcome.Nurs Stand.1992;7(11 Suppl QA):45.Available

24. Huo X P., Sun H., Zhu H W., et al. An evidence-based study on fall prevention in elderly inpatients [J]. Chinese Journal of Modern nursing. 2016,22(05):613-617.

25. National health and Family Planning Commission Official Interpretation $\square$ Measures for quality control of medical treatment囚[J]. China Health Quality Management, 2016,23(z2):1-2. 
26. Hendrich A.How to try this: predicting patient falls. Using the Hendrich II Fall Risk Model in clinical practice.AM J NURS.2007;107(11):50-58, 58-59.Available from:http://dx.doi.org/10.1097/01.NAJ.0000298062.27349.8e.

27. Morse JM, Black C, Oberle K, Donahue P.A prospective study to identify the fall-prone patient.SOC SCI MED.1989;28(1):81-86.Available from:http://dx.doi.org/10.1016/0277-9536(89)90309-2.

28. Ocker SA, Barton SA, Bollinger N, Leaver CA, Harne-Britner S, Heuston MM.Preventing Falls Among Behavioral Health Patients.AM J NURS.2020;120(7):61-68.Available from:http://dx.doi.org/10.1097/01.NAJ.0000688256.96880.a3.

29. Staggs VS, Knight JE, Dunton N.Understanding unassisted falls: effects of nurse staffing level and nursing staff characteristics.J NURS CARE QUAL.2012;27(3):194-199.Available from:http://dx.doi.org/10.1097/NCQ.0b013e318241da2d.

30. Avin KG, Hanke TA, Kirk-Sanchez N, McDonough CM, Shubert TE, Hardage J, Hartley G.Management of falls in community-dwelling older adults: clinical guidance statement from the Academy of Geriatric Physical Therapy of the American Physical Therapy Association.PHYS THER.2015;95(6):815-834.Available from:http://dx.doi.org/10.2522/ptj.20140415.

31. Johnson M, Kelly L, Siric K, Tran DT, Overs B.Improving falls risk screening and prevention using an e-learning approach.J Nurs Manag.2015;23(7):910-919.Available from:http://dx.doi.org/10.1111/jonm.12234.

32. Ballesteros MF, Webb K, McClure RJ.A review of CDC's Web-based Injury Statistics Query and Reporting System (WISQARS): Planning for the future of injury surveillance. J SAFETY RES.2017;61:211-215.Available from:http://dx.doi.org/10.1016/j.jsr.2017.01.001.

33. Heng H, Jazayeri D, Shaw L, Kiegaldie D, Hill AM, Morris ME. Educating hospital patients to prevent falls: protocol for a scoping review. BMJ OPEN.2019;9(9):e30952.Available from:http://dx.doi.org/10.1136/bmjopen-2019-030952.

34. Stevens JA, Phelan EA. Development of STEADI: a fall prevention resource for health care providers. Health Promot Pract.2013;14(5):706-714.Available from:http://dx.doi.org/10.1177/1524839912463576.

35. Radecki B, Keen A, Miller J, McClure JK, Kara A. Innovating Fall Safety: Engaging Patients as Experts.J NURS CARE QUAL.2020;35(3):220-226.Available from:http://dx.doi.org/10.1097/NCQ.0000000000000447.

36. Moe K, Brockopp D, McCowan D, Merritt S, Hall B.Major Predictors of Inpatient Falls: A Multisite Study.J Nurs Adm.2015;45(10):498-502.Available from:http://dx.doi.org/10.1097/NNA.0000000000000241.

37. Cameron ID, Dyer SM, Panagoda CE, Murray GR, Hill KD, Cumming RG, Kerse N. Interventions for preventing falls in older people in care facilities and hospitals. Cochrane Database Syst Rev.2018;9:D5465.Available from:http://dx.doi.org/10.1002/14651858.CD005465.pub4.

38. Haines TP, Bennell KL, Osborne RH, Hill KD. Effectiveness of targeted falls prevention programme in subacute hospital setting: randomised controlled trial.BMJ.2004;328(7441):676.Available from:http://dx.doi.org/10.1136/bmj.328.7441.676.

39. Blain H, Dabas F, Mekhinini S, Picot MC, Miot S, Bousquet J, Boubakri C, Jaussent A, Bernard PL. Effectiveness of a programme delivered in a falls clinic in preventing serious injuries in high-risk older adults: A pre- and postintervention study.MATURITAS.2019;122:80-86.Available from:http://dx.doi.org/10.1016/j.maturitas.2019.01.012. 